\title{
Nuclear Resonant Scattering of Synchrotron Radiation: Applications in Magnetism of Layered Structures
}

\author{
Kai Schlage and Ralf Röhlsberger \\ Deutsches Elektronen Synchrotron DESY, Notkestr. 85, 22607 Hamburg, Germany
}

\begin{abstract}
Nuclear resonant scattering of synchrotron radiation has become an established tool within condensed-matter research. Synchrotron radiation with its outstanding brilliance, transverse coherence and polarization has opened this field for many unique studies, for fundamental research in the field of light-matter interaction as well as for materials science. This applies in particular for the electronic and magnetic structure of very small sample volumes like microand nanostructures and samples under extreme conditions of temperature and pressure. This article is devoted to the application of the technique to nanomagnetic systems such as thin films and multilayers. After a basic introduction into the method, a number of our experiments are presented to illustrate how magnetic spin structures within such layer systems can be revealed.
\end{abstract}

\section{INTRODUCTION}

Atomic nuclei can be very sensitive probes of condensed matter properties. A number of spectroscopic techniques have been developed to probe condensed matter via nuclear hyperfine interactions, constituting the field of nuclear condensed matter physics [1,2]. A very prominent method in this field is Mössbauer spectroscopy that probes internal magnetic and electric fields in the sample by analysing the energetic hyperfine splitting of the nuclear levels via absorption of $\gamma$-radiation. Traditionally, this technique is applied in the energy domain where the radiation from a monochromatic radioactive source is Doppler tuned to measure the absorption spectrum around the nuclear resonance with nano-eV resolution. The hyperfine interaction of the nuclei in the sample typically lifts the degeneracy of the nuclear levels that manifests as a splitting and/or broadening of the absorption line(s).

Synchrotron radiation effectively allows one to perform the Fourier transform of Mössbauer spectroscopy from the energy domain into the time domain: The radiation is energetically broad (in contrast to the mono-energetic $\gamma$-radiation emitted by a radioactive source), and it comes in pulses with a duration of $50-100$ ps (in contrast to the continuously emitting radioactive source). Thus, these pulses excite all hyperfine-split resonances in the sample at the same time. In the subsequent coherent decay the interference of the emitted waves leads to a temporal beat pattern, very similar to the temporal acoustic beats of slightly-detuned tuning forks. From such a beat pattern the hyperfine interaction parameters of the nuclei in the sample can be deduced.

Within the natural linewidth of the Mössbauer transition, the brilliance of synchrotron radiation sources exceeds that of radioactive sources by several orders of magnitude. This was realized early by Ruby [3] and the first observation of the time-based analogue of Mössbauer spectroscopy a.k.a. nuclear resonant scattering of synchrotron radiation was reported by Gerdau et al. [4] in Bragg scattering geometry and later by Hastings et al. [5] in forward scattering geometry. Since then, the method rapidly developed and is now available 
at many $3^{\text {rd }}$-generation synchrotron sources (ESRF, APS, Spring8, PETRA III) around the world, covering a broad range in all fields of the natural sciences $[2,6]$.

\section{BASIC PRINCIPLES OF NUCLEAR RESONANT SCATTERING}

Due to the narrow nuclear resonance width in the range of neV $-\mu \mathrm{eV}$, the scattering process takes place on time scales ranging from $\mu$ s to ns. This allows for a discrimination of the resonantly scattered radiation from the non-resonant charge scattering and fluorescence that proceeds on time scales below $10^{-15} \mathrm{~s}$. A synopsis of the method of time-resolved coherent nuclear resonant scattering is given in Fig.1.

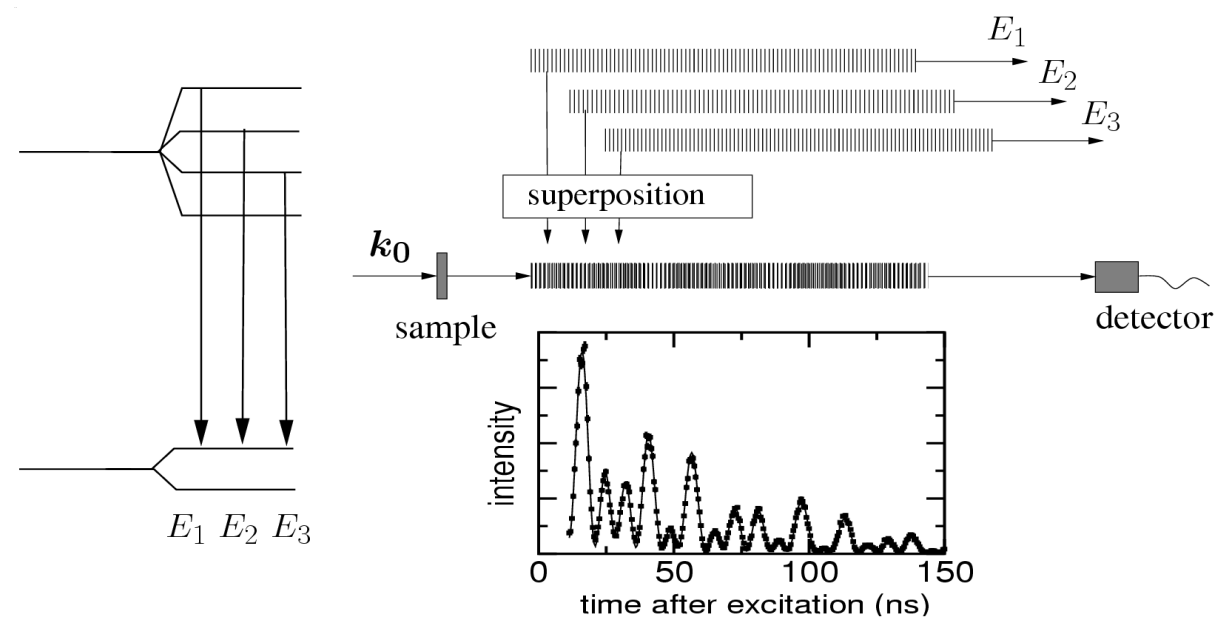

Fig.1 Principle of coherent nuclear resonant scattering in forward direction. Transitions between nuclear levels are excited simultaneously by synchrotron radiation pulses at $t=0$. In this example, three selected waves with slightly different frequencies are emitted from the decaying nuclei in the sample. Their superposition leads to quantum beats in the temporal evolution of the decay. This is illustrated here by overlaying three wavetrains with slightly different spatial frequencies, leading to a Moiré pattern. The intensity modulation of this pattern corresponds to the temporal quantum beats that are detected with a time-resolving detector like an avalanche photodiode (APD).

The transmission of $\mathrm{x}$-rays through a slab of material that contains resonant nuclei can be described in a straightforward fashion via the energy-dependent index of refraction $n(\omega)$ :

$$
A(z, \omega)=\exp \left[\operatorname{in}(\omega) k_{0} z\right] A_{0} .
$$

which gives the amplitude of the radiation field in depth $\mathrm{z}$ of the sample. $A_{0}$ is the amplitude of the incident radiation field. The index of refraction $n$ is related to the forward scattering length $M$ via

$$
n(\omega)=1+\frac{2 \pi}{k_{0}^{2}} \sum_{k} \rho_{k} M_{k}(\omega),
$$

with $\rho_{k}$ being the number density of the $k^{\text {th }}$ atomic species in the sample. These equations can be generalized to a multitude of scattering problems, ranging from simple forward scattering to anisotropic optics, thin-film reflection and Bragg diffraction from crystals and 
gratings [9]. The number $N$ of open scattering channels determines the dimension of $M$, i.e., $M$ is a scalar quantity for isotropic (polarisation-independent) forward scattering, a $2 \times 2$ matrix for anisotropic (polarisation-dependent) forward scattering, a $4 \times 4$ matrix for anisotropic (2-beam) Bragg diffraction which includes also nuclear resonant scattering in grazing incidence geometry. Correspondingly, the index of refraction $n(\omega)$ is a $\mathrm{N}$ dimensional matrix.

Correspondingly, the quantities $\mathrm{A}_{0}$ and $\mathrm{A}(\mathrm{z})$ are vectors with $N$ components that describe the amplitudes of the radiation field in the $N$ scattering channels. This formalism naturally contains all multiple scattering processes within the sample, i.e., the resulting radiation field $\mathrm{A}(\mathrm{z})$ is a self-consistent solution to the scattering problem within the dynamical theory of $\mathrm{x}-$ ray scattering.

The specific interaction of the photons with the atoms in the material is contained in the scattering length $M$. It is well known that optical properties of a system change drastically if the photon energy approaches an atomic resonance. This is valid for the complete spectral range from the infrared into the hard x-ray regime. The remarkable features of $x$-ray scattering from inner-shell resonances have been discovered and exploited when highbrilliance synchrotron radiation became available $[10,11]$. This applies for $\mathrm{x}$-ray scattering from nuclear resonances as well [4,6,12].

Fig. 2 shows the real and imaginary parts of the forward scattering amplitude of ${ }^{57} \mathrm{Fe}$ over an energy range from $6 \mathrm{keV}$ to $16 \mathrm{keV}$. Around $7 \mathrm{keV}$ one observes the electronic K-edge of the material as it results from excitation of an electron in the K-shell into the continuum. At a photon energy of $14.4 \mathrm{keV}$ one observes an extremely sharp feature that results from resonant excitation of the ${ }^{57} \mathrm{Fe}$ nucleus. The shape of this resonance becomes apparent only if one blows up the energy scale to the neV regime. The vertical axis is scaled in units of the classical electron radius $r_{0}$ which is the scattering length of a single electron. Thus it appears that near the resonance the scattering strength of the nucleus corresponds to an atom with $\mathrm{Z}$ $=200$ ! This means that out of the small energy range around the resonance a very strong scattering signal can arise that exceeds that of electronic resonances by far [2].

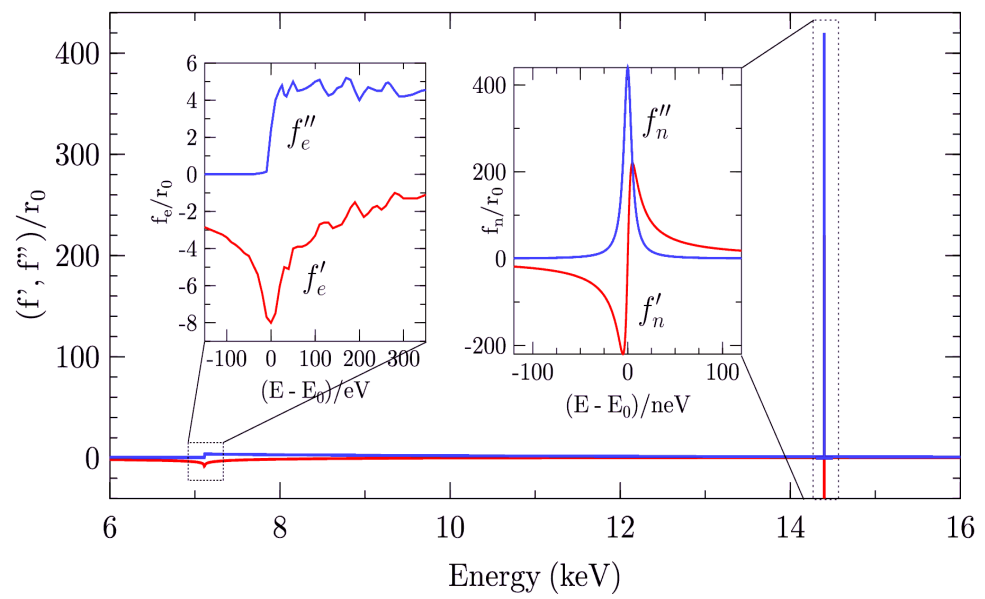

Fig. 2: Anomalous scattering lengths (real part $\mathrm{f}^{\prime}$ and imaginary part $\mathrm{f}^{\prime \prime}$ ) for ${ }^{57} \mathrm{Fe}$ including the K-edge around $7 \mathrm{keV}$ and the nuclear resonance around $14.4 \mathrm{keV}$, measured in units of the free electron radius $\mathrm{r}_{0}$. While around the K-edge the anomalous contributions amount to only a few electrons, around the nuclear resonance they correspond to more than 200 electrons!

Near the resonance the scattering amplitude of an atom with a single nuclear resonance can be written as 


$$
M(\omega)=E(\omega)+N(\omega),
$$

where $E$ and $N$ are the electronic and nuclear contributions to the scattering length, respectively:

$$
\begin{gathered}
{[E(\omega)]_{\mu v}=\left(\boldsymbol{\varepsilon}_{\mu} \cdot \varepsilon_{v}\right)\left[-Z r_{0}+f_{e}{ }^{\prime}+i f_{e^{\prime \prime}}{ }^{\prime}\right]=\left(\boldsymbol{\varepsilon}_{\mu} \cdot \boldsymbol{\varepsilon}_{v}\right)\left[-Z r_{0}+f_{e}{ }^{\prime}+i \frac{k_{0}}{4 \pi} \sigma(\omega)\right]} \\
{[N(\omega)]_{u v}=\left(\boldsymbol{\varepsilon}_{\mu} \cdot \boldsymbol{\varepsilon}_{V}\right)\left[f_{n}{ }^{\prime}+i f_{n}{ }^{\prime \prime}\right]=\left(\boldsymbol{\varepsilon}_{\mu} \cdot \boldsymbol{\varepsilon}_{v}\right) \frac{f_{0}}{x+i}=\left(\boldsymbol{\varepsilon}_{\mu} \cdot \boldsymbol{\varepsilon}_{v}\right)\left[\frac{f_{0} x}{x^{2}+1}+i \frac{f_{0}}{x^{2}+1}\right]}
\end{gathered}
$$

where $Z$ is the atomic number, $r_{0}$ the classical electron radius, and $\sigma$ is the total absorption cross section. $\left(\varepsilon_{\mu}, \varepsilon_{v}\right)$ are the vectors of the polarization basis. Their scalar product that appears in the expressions above indicates that the scattering process conserves the polarization, i.e., the $2 \times 2$ matrices $E$ and $N$ are diagonal. This is in general not the case if the nucleus is subject to an electric or magnetic hyperfine interaction, as we shall see below.

The nuclear scattering length $N(\omega)$ is given here for a single, unsplit resonance line (i.e., no hyperfine interaction). $x=2\left(E-E_{0}\right) / \Gamma_{0}$ denotes the deviation of the energy from the exact resonance energy $E_{0}$ measured in units of the natural linewidth $\Gamma_{0}$ of the transition. $f_{0}$ expresses the oscillator strength of the nuclear resonance:

$$
f_{0}=\frac{f_{L M}}{2 k_{0}} \frac{2 I_{e}+1}{2 I_{g}+1} \frac{1}{1+\alpha}
$$

where $f_{L M}$ is the Lamb-Mössbauer factor that describes the relative fraction of photons that are elastically scattered (i.e., without recoil). $I_{e}$ and $I_{g}$ are the spins of the ground and excited nuclear state, respectively, and $\alpha$ is the coefficient of internal conversion.

For the $14.4 \mathrm{keV}$ transition of ${ }^{57} \mathrm{Fe}$ we have $\alpha=8.2, I_{g}=1 / 2, I_{e}=3 / 2$ and $k_{0}=7.3 \times 10^{10} \mathrm{~m}^{-1}$. In $\alpha$-Fe at room temperature we have $f_{L M} \approx 0.8$ so that $f_{0} \approx 1.2 \cdot 10^{-12} \mathrm{~m}$. In the case of magnetic materials, a finite magnetic hyperfine field acts on the resonant nuclei. In ferromagnetic $\alpha-\mathrm{Fe}$, for example, the magnitude of this field is $33.3 \mathrm{~T}$ at room temperature. As a consequence, the degeneracy of nuclear levels with spin $I_{N} \geq 1 / 2$ is lifted, i.e. these states split into $2 I_{N}+1$ magnetic sublevels between which electromagnetic transitions can occur according to the multipole selection rules. For an electric dipole transition the nuclear scattering length reads:

$$
[N(\omega)]_{\mu v}=\frac{3}{16 \pi}\left\{\begin{array}{l}
\left(\varepsilon_{\mu} \cdot \varepsilon_{v}\right)\left[F_{+1}+F_{-1}\right] \\
-i\left(\varepsilon_{\mu} \times \varepsilon_{v}\right) \cdot \mathbf{m}\left[F_{+1}-F_{-1}\right] \\
+\left(\varepsilon_{\mu} \cdot \mathbf{m}\right)\left(\varepsilon_{v} \cdot \mathbf{m}\right)\left[2 F_{0}-F_{+1}-F_{-1}\right]
\end{array}\right\}
$$

The quantities $F_{0}=F_{0}(\omega)$ and $F_{ \pm}=F_{ \pm}(\omega)$ are functions that describe the energy dependence of the scattering length for the transitions with change of magnetic quantum number $\Delta m=0, \pm 1$ [13]. In case of a magnetic dipole transition (as for the $14.4 \mathrm{keV}$ transition of ${ }^{57} \mathrm{Fe}$ ) the role of electric and magnetic fields of the radiation are interchanged. Then one has to transform the polarization vectors in the expression above via $\varepsilon \rightarrow \varepsilon \times k_{0}$.

The scalar and vector products involving the polarization basis vectors $\varepsilon_{\mu}$ and $\varepsilon_{v}$ as well as the unit vector $\mathbf{m}$ in the direction of the local magnetization (corresponding to the direction 
of the local hyperfine field at the position of the nucleus) indicate a strong polarization dependence of the scattering process. The three terms in the equation above represent different polarization dependencies. The first term is not sensitive to the sample magnetization. Its polarization dependence given by $\varepsilon_{\mu} \cdot \varepsilon_{v}$ is that of non-resonant charge scattering. The second term describes circular dichroism because it depends on the difference between the resonant scattering amplitudes $F_{+}$and $F_{-}$. Since its polarization dependence is $\varepsilon_{\mu} \times \varepsilon_{v}$, it describes orthogonal scattering, e.g., $\sigma \rightarrow \pi$ and $\pi \rightarrow \sigma$. The third term describes linear magnetic dichroism. Its polarization dependence allows for all scattering processes within the given polarization basis. Due to the very high sensitivity of the nuclear scattering length to the magnetization direction relative to the incident wave vector, nuclear resonant scattering constitutes a very sensitive probe of local spin structures in magnetic materials. With this formalism one is now able to calculate the index of refraction in the vicinity of the nuclear resonance. The index of refraction can then be used in any optical code, e.g., Fresnel formulas for single interfaces or multibeam interference equations for calculating nuclear resonant reflectivities from thin films and multilayers [9].

To illustrate the sensitivity of the method to the magnetization direction within the sample we have calculated the time dependence of nuclear resonant scattering for selected magnetization geometries in Fig. 3 .

\begin{tabular}{|c|c|c|c|}
\hline & Geometry & Scattering matrix & Time spectrum \\
\hline$A$ & $m \mathrm{k}_{0}$ & $\left\{\begin{array}{cc}F_{+1}+F_{-1} & -i\left(F_{+1}-F_{-1}\right) \\
i\left(F_{+1}-F_{-1}\right) & F_{+1}+F_{-1}\end{array}\right.$ & \\
\hline$B$ & & $\left(\begin{array}{cc}F_{+1}+F_{-1} & 0 \\
0 & F_{+1}+F_{-1}\end{array}\right)$ & \\
\hline C & 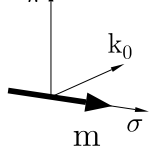 & $\left(\begin{array}{cc}F_{+1}+F_{-1} & 0 \\
0 & 2 F_{0}\end{array}\right)$ & \\
\hline$D$ & $\underbrace{\pi}_{\sigma}$ & $\left(\begin{array}{cc}2 F_{0} & 0 \\
0 & F_{+1}+F_{-1}\end{array}\right)$ & \\
\hline
\end{tabular}

Fig. 3: Time spectra of nuclear resonant scattering for selected orientations of the magnetic hyperfine field $m$ relative to the incident wave vector $k_{0}$. The $2 \times 2$ matrix of the nuclear scattering length is given in a linear polarization basis $(\sigma, \pi)$. The time spectra are calculated for a $2 \mathrm{~nm}$ thick, ferromagnetic $\alpha-{ }^{57} \mathrm{Fe}$ film on a tungsten substrate, assuming purely $\sigma$ polarized incident radiation and unpolarized detection. This is the most frequently used scattering geometry in experiments with synchrotron radiation. A, C, D display the results for a unidirectional magnetization of the sample. B results from the superposition of two magnetic sublattices in antiparallel alignment as in antiferromagnets, for example.

One clearly realizes that the time spectra of the nuclear resonant reflectivity can be used as fingerprints of the magnetic structure in the sample, although some care has to be taken since there are different magnetic configurations that lead to the same time spectrum. To rule out such ambiguities, it is recommended to record time spectra for at least two different orientations of the sample relative to the beam [15]. 
While most of the experiments to date have been done with ${ }^{57} \mathrm{Fe}$, this is not the only isotope that has been employed in nuclear resonant scattering. Table 1 provides a list of these isotopes, whereby ${ }^{121} \mathrm{Sb}[16]$ and ${ }^{125} \mathrm{Te}$ [17] are the most recent additions.

Table 1 Parameters of Mössbauer isotopes that have been used in experiments with synchrotron radiation to date (Sept. 2012). $\alpha$ is the natural abundance of the isotope, $I_{g}$ and $I_{e}$ are the spins of the ground and excited state, respectively, $\Gamma_{\mathrm{o}}$ is the natural linewidth, and $\tau_{\mathrm{o}}$ is the lifetime.

\begin{tabular}{cccccccc}
\hline Isotope & $\begin{array}{c}E_{\gamma} \\
{[\mathrm{keV}]}\end{array}$ & $\begin{array}{c}\mathrm{a} \\
{[\%]}\end{array}$ & $\begin{array}{c}\Gamma_{\mathrm{o}} \\
{[\mathrm{neV}]}\end{array}$ & $\begin{array}{c}\tau_{\mathrm{o}} \\
{[\mathrm{ns}]}\end{array}$ & $I_{g}$ & $I_{e}$ & $\begin{array}{c}\text { multi- } \\
\text { polarity }\end{array}$ \\
\hline \hline${ }^{181} \mathrm{Ta}$ & 6.23 & 99.9 & 0.067 & 9870 & $7 / 2$ & $9 / 2$ & $\mathrm{E} 1$ \\
${ }^{169} \mathrm{Tm}$ & 8.41 & 100 & 114 & 5.8 & $1 / 2$ & $3 / 2$ & $\mathrm{M} 1$ \\
${ }^{83} \mathrm{Kr}$ & 9.40 & 12.0 & 3.3 & 212 & $9 / 2$ & $7 / 2$ & M1 \\
${ }^{73} \mathrm{Ge}$ & 13.28 & 7.8 & 0.15 & 4260 & $9 / 2$ & $5 / 2$ & $\mathrm{E} 2$ \\
${ }^{57} \mathrm{Fe}$ & 14.41 & 2.1 & 4.7 & 141 & $1 / 2$ & $3 / 2$ & M1 \\
${ }^{153} \mathrm{Eu}$ & 21.53 & 47.8 & 47.0 & 14.1 & $5 / 2$ & $7 / 2$ & M1 \\
${ }^{149} \mathrm{Sm}$ & 22.49 & 13.8 & 64.1 & 10.3 & $7 / 2$ & $4 / 2$ & M1 \\
${ }^{119} \mathrm{Sn}$ & 23.87 & 8.6 & 25.7 & 25.7 & $1 / 2$ & $3 / 2$ & M1 \\
${ }^{201 \mathrm{Hg}}$ & 26.27 & 13.2 & 760 & 0.9 & $3 / 2$ & $1 / 2$ & $\mathrm{M} 1$ \\
${ }^{161} \mathrm{Dy}$ & 26.65 & 18.9 & 16.2 & 40.8 & $5 / 2$ & $5 / 2$ & $\mathrm{E} 1$ \\
${ }^{40} \mathrm{~K}$ & 29.83 & 0.0117 & 160.5 & 4.13 & 4 & 3 & M1 \\
${ }^{125} \mathrm{Te}$ & 35.49 & 7.0 & 310 & 2.14 & $1 / 2$ & $3 / 2$ & M1 \\
${ }^{121} \mathrm{Sb}$ & 37.13 & 57.25 & 130.0 & 5.0 & $5 / 2$ & $7 / 2$ & M1 \\
${ }^{61} \mathrm{Ni}$ & 67.40 & 1.25 & 88.3 & 7.5 & $3 / 2$ & $5 / 2$ & M1 \\
\hline
\end{tabular}

\section{Imaging the Magnetic Spin Structure of Exchange-Spring Magnetic Layers}

The isotopic sensitivity of nuclear resonant scattering enables one to probe selected parts of a layer system by depositing ultrathin layers of a Mössbauer isotope. Replacing an element with its Mössbauer isotope (e.g., Fe by ${ }^{57} \mathrm{Fe}$ ) does not disturb the chemical integrity of the layer system. Precise control over the isotopic probe layer thickness thus enables one to reach atomic resolution in the determination of magnetic properties. This is particularly interesting for the study of magnetic heterostructures where the magnetic properties critically depend on the interfacial structure. Prominent examples are nanocomposites that consist of exchange-coupled soft- and hard-magnetic phases. Thin bilayers consisting of a hard-and a soft-magnetic material are ideal model systems to investigate the fundamental properties of this coupling mechanism. As a characteristic property of such systems, the magnetization of the soft-magnetic film at the interface is pinned to the hard-magnetic film as a result of the exchange interaction. With increasing distance from the interface, the exchange coupling becomes weaker and the magnetization may rotate under the action of an external field. If, for example, the external field is applied orthogonal to the magnetization direction of the hard layer, the magnetic moments in the soft layer arrange in a spiral structure along the normal, as shown schematically in Fig. 4. Because of the reversible nature of this rotation, this is called the exchange-spring effect [19].

Here we show how to apply nuclear resonant scattering from ultrathin probe layers of ${ }^{57} \mathrm{Fe}$ to directly measure the in-depth magnetic structure of exchange-spring bilayers [22]. The sample was a bilayer consisting of $11 \mathrm{~nm}$ soft-magnetic Fe on $30 \mathrm{~nm}$ hard-magnetic FePt. To minimize oxidation of the Fe layer, it was coated with a $3 \mathrm{~nm}$ thick Ag layer. All the layers were prepared by RF-magnetron sputtering in a high-vacuum system with a base pressure of $2 \times 10^{-7}$ mbar. The FePt layer was produced by co-sputtering of Fe and Pt to obtain a composition close to $\mathrm{Fe}_{55} \mathrm{Pt}_{45}$. Subsequent annealing of this layer resulted in the formation of the hardmagnetic $\mathrm{L} 1_{0}$ phase with a coercivity of $0.95 \mathrm{~T}$ at room temperature. An ultrathin inclined probe layer of ${ }^{57} \mathrm{Fe}$ was embedded within the Fe layer, see Fig. 4 . The sample was 
mounted in a cryomagnet system and exposed to an external field of about $3.5 \mathrm{~T}$ to saturate the FePt and to introduce a remanent uniaxial magnetization along the direction of the incident wave vector $k_{0}$. During the measurements, the sample was cooled to $4.2 \mathrm{~K}$ and subjected to variable external fields $H$ perpendicular to the beam direction. To measure the depth dependence of the spin rotation, the sample was displaced transversely to the beam. Time spectra taken at various positions $\Delta x$ are shown in Fig. 5 for an external field of $H=$ $160 \mathrm{mT}$.

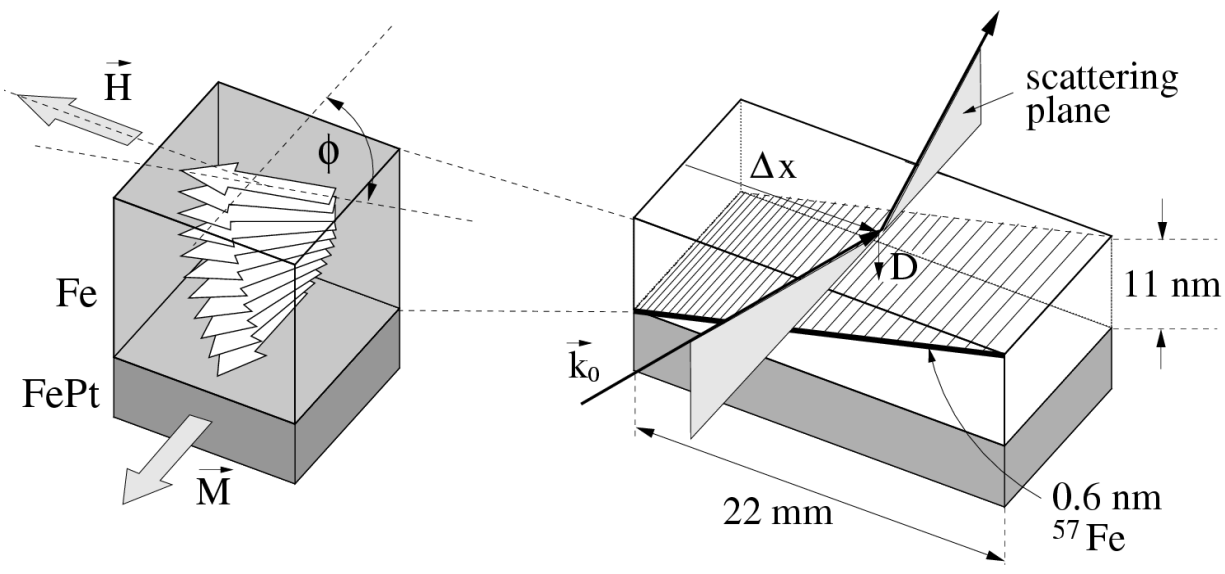

Fig.4 Left: Spin structure in an exchange-spring bilayer consisting of a soft-magnetic layer (Fe) on a hard-magnetic layer $(\mathrm{FePt})$ with unidirectional anisotropy. An external field $H$ is applied perpendicular to the remanent magnetization $M$ of the hard layer. Right: To image the resulting magnetic spiral, an inclined ultrathin probe layer of ${ }^{57} \mathrm{Fe}$ is deposited within the soft layer. The magnetic properties in depth $D$ of the film are probed by adjusting the transverse displacement $\Delta x$ of the sample relative to the scattering plane [22]. The polarization of the incident beam is perpendicular to the scattering plane.

The characteristic changes in the beat pattern reflect the rotation of the magnetization from an almost parallel alignment at $\Delta \mathrm{x}=0 \mathrm{~mm}$ towards increasing perpendicular orientation with increasing $\Delta \mathrm{x}$ : This corresponds to a gradual transition from geometry $\mathrm{A}$ to geometry $\mathrm{C}$ in Fig. 3. From each of these spectra the in-plane rotation angle $\varphi$ of the magnetization was deduced by a fit of the data (solid lines). The resulting depth dependence of the rotation angle is shown in Fig. 5 for external fields of 160, 240 and $500 \mathrm{mT}$.

Dashed lines are simulations according to a one-dimensional micromagnetic model as outlined in [22], assuming the magnetic properties to be homogeneous throughout the Fe layer. Close to the Fe/Ag interface, however, the simulation clearly deviates from the measured data. This can be explained by a reduction of the exchange constant in the top 3 $\mathrm{nm}$ of the Fe layer to $1 / 3$ of the value in the main body of the Fe layer. The solid lines are corresponding simulations, yielding a very good description of the experimental data. This example demonstrates how ultrathin isotopic probe layers can be employed to determine the depth-dependent magnetic spin structure in exchange-coupled thin films with sub-nm resolution. See also studies on the system $\mathrm{Fe} / \mathrm{Cr} / \mathrm{Fe}$ [14]. In one of the next examples we show how NRS can be applied to study magnetic ordering in multilayers. 

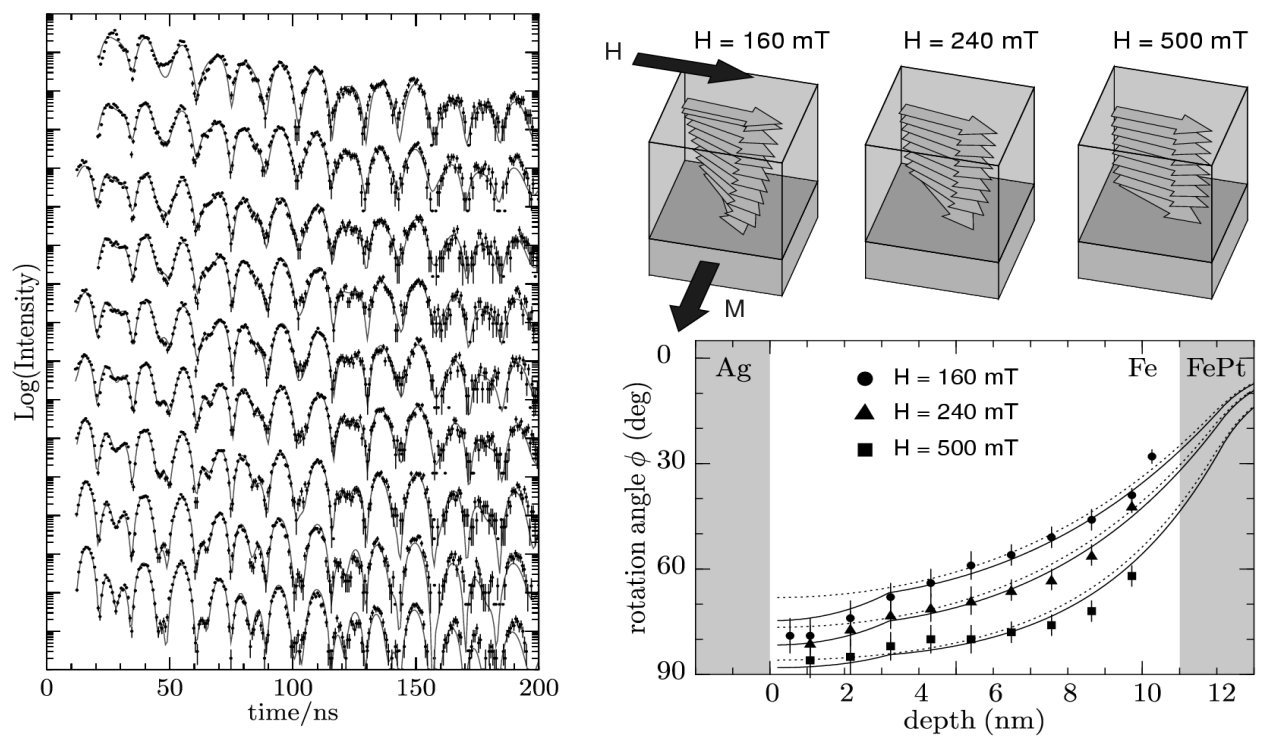

Fig. 5: Measured time spectra of nuclear resonant grazing incidence reflection from the $\mathrm{Fe} / \mathrm{FePt}$ exchange-spring system described in the text for various lateral positions starting from $\Delta x=2 \mathrm{~mm}$ (top) to $\Delta \mathrm{x}=18 \mathrm{~mm}$ (bottom) in a perpendicular magnetic field of $\mathrm{H}=160 \mathrm{mT}$. The solid lines are fits to the data with the rotation angle $\varphi$ as the only free parameter. From such measurements the depth dependence of the magnetization can be determined with very high spatial resolution, as shown in the right column for various external magnetic fields.

\section{Antiferromagnetic Coupling in Fe/Cr Multilayers}

Antiferromagnetic multilayers can be used as model systems to study the behavior of finite magnetic spin systems. Here we choose the archetypal Fe/Cr multilayer as an example. In such multilayers the adjacent Fe layers are exchange coupled through the non-magnetic $\mathrm{Cr}$ spacer in which standing waves of spin polarized conduction electrons mediate the antiferromagnetic coupling $[35,36]$. The coupling is an oscillatory function of the $\mathrm{Cr}$ spacer layer thickness. At a $\mathrm{Cr}$ thickness of $\mathrm{d}=1.3 \mathrm{~nm}$ one observes the maximum of the antiferromagnetic (afm) coupling strength. Due to the afm coupling of the Fe layers, the magnetic unit cell is twice as large as the chemical unit cell. Probing such a structure with a spin-sensitive scattering method leads to half-order Bragg reflections that are located between the Bragg peaks of the chemical structure [30]. Nuclear resonant scattering of synchrotron radiation exhibits a similar sensitivity to magnetic superstructures as neutron scattering does [27]. The very high brilliance of modern synchrotron radiation sources, however, leads to particular advantages of the NRS technique like high spatial resolution via micro focusing and short data acquisition times in the range of a few minutes for a reflectivity scan.

The Fe/Cr multilayer in this example consisted of 30 bilayers of $(1.9 \mathrm{~nm} \mathrm{Fe} / 1.5 \mathrm{~nm} \mathrm{Cr})$ on a $\mathrm{Si}$ wafer, produced by sputter deposition in an Argon atmosphere from a $\mathrm{Cr}$ target and a $\mathrm{Fe}$ target enriched to $95 \%$ in ${ }^{57} \mathrm{Fe}$. The angular dependence of the electronic and nuclear reflectivity is shown in Fig. 6. The chemical periodicity of the multilayer with a period of 3.4 $\mathrm{nm}$ results in the first-order electronic Bragg reflection at a grazing angle of $13 \mathrm{mrad}$. The same peak is seen also in the nuclear reflectivity. However, in contrast to the electronic reflectivity, the nuclear reflectivity (Fig. 6c) shows peaks also at the angular positions of the half-order reflections, corresponding to a spatial period of $6.8 \mathrm{~nm}$. This is the period of a 
magnetic superstructure that results from the antiferromagnetic coupling of the neighboring iron layers.
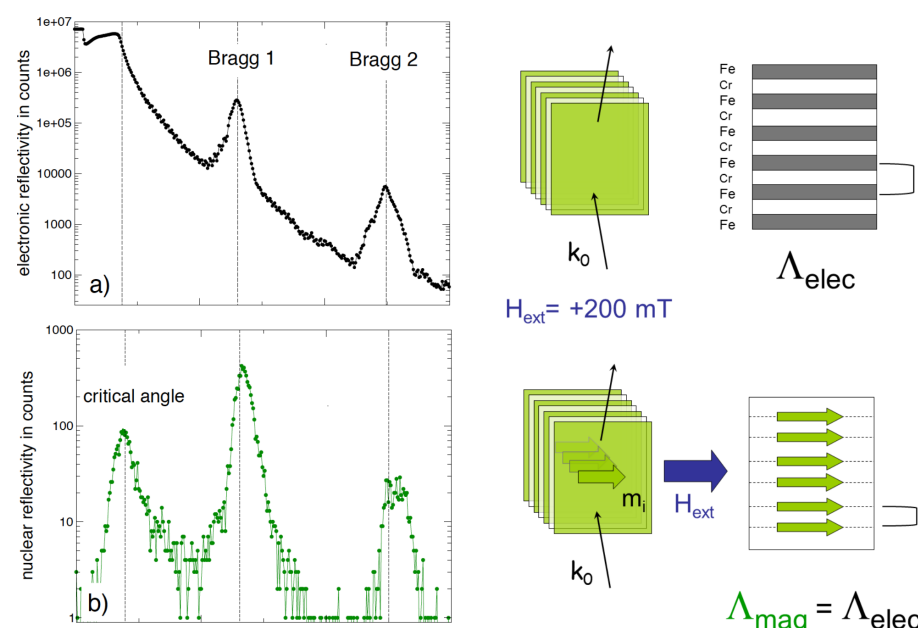

$\mathrm{H}_{\mathrm{ext}}=+200 \mathrm{mT}$

$$
\Lambda_{\text {elec }}
$$
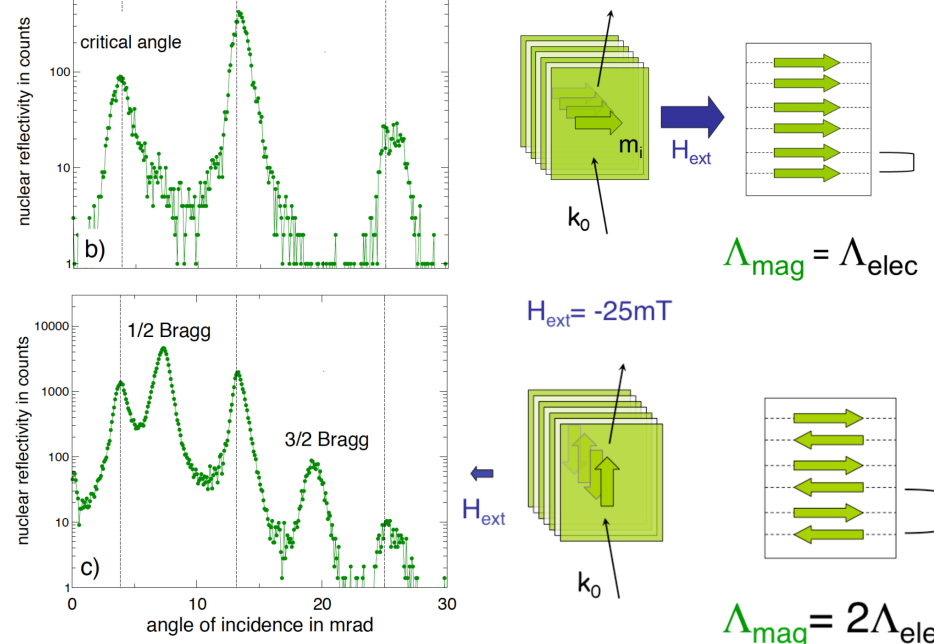

$H_{\text {ext }}=-25 m T$
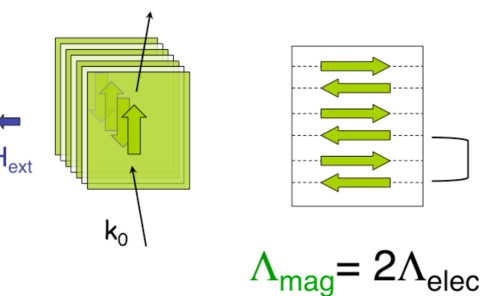

Fig. 6: Electronic and nuclear reflectivity of a $\left[{ }^{57} \mathrm{Fe}(1.9 \mathrm{~nm}) / \mathrm{Cr}(1.5 \mathrm{~nm})\right]_{30}$ multilayer structure. (a) The electronic reflectivity is characterized by strong Bragg peaks due to the structural periodicity of system. In the nuclear reflected channel $(b, c)$ an additional peak appears close to the critical angle. Its results from the high field intensity of the evanescent wave close to the surface that forms at the critical angle. (b) In the magnetically saturated sample $\left(B_{\text {ext }}=200 \mathrm{mT}\right)$ the ferromagnetic alignment of the iron layers causes a magnetic periodicity which is the same as the electronic one. (c) At low field the antiferromagnetic alignment of the iron causes a magnetic periodicity of the system with twice the period of the structural one $\left(\Lambda_{\text {mag }}=2 \Lambda_{\text {elec }}\right)$. As a result, a magnetic superstructure reflection appears with highest intensity for an antiferromagnetic arrangement collinear to $k_{0}$.

Fig. $6 \mathrm{~b}$ shows the nuclear refelectivity of the same multilayer in the magnetic saturation regime $\left(\mathrm{H}_{\mathrm{ext}}=200 \mathrm{mT}\right)$. The parallel alignment of the iron layers and the elimination of the magnetic superstructure results in vanishing superstructure Bragg peaks. The suppression of the nuclear intensity at the half-order reflections reaches more than $95 \%$ and highlights the sensitivity of this method to study the field dependent magnetic configuration in the multilayer structure.

\section{Spin-flop transition}

The simulation of the nuclear reflectivity can be used to elaborate the vectorial magnetic depth profile of the coupled iron layers. The high count rate of the superstructure reflections (up to several $\mathrm{kHz}$ of resonantly scattered photons) can in addition be used to detect field dependent magnetization curves. Its intensity is a measure of the projection of the antiferromagnetically coupled magnetic sublattices onto the direction of the incoming synchrotron beam with wavevector $\mathrm{k}_{0}$. Its field dependence allows to identify the magnetic saturation behavior of the multilayer structure (Fig. 7) and can also be used to study 
magnetic reorientation phenomena which are not accompanied by a significant change in the integral net magnetization of the system.

Such a phenomenon can be observed in antiferromagnetically coupled multilayers with an even number of magnetic layers if an increasing external magnetic field is applied along the

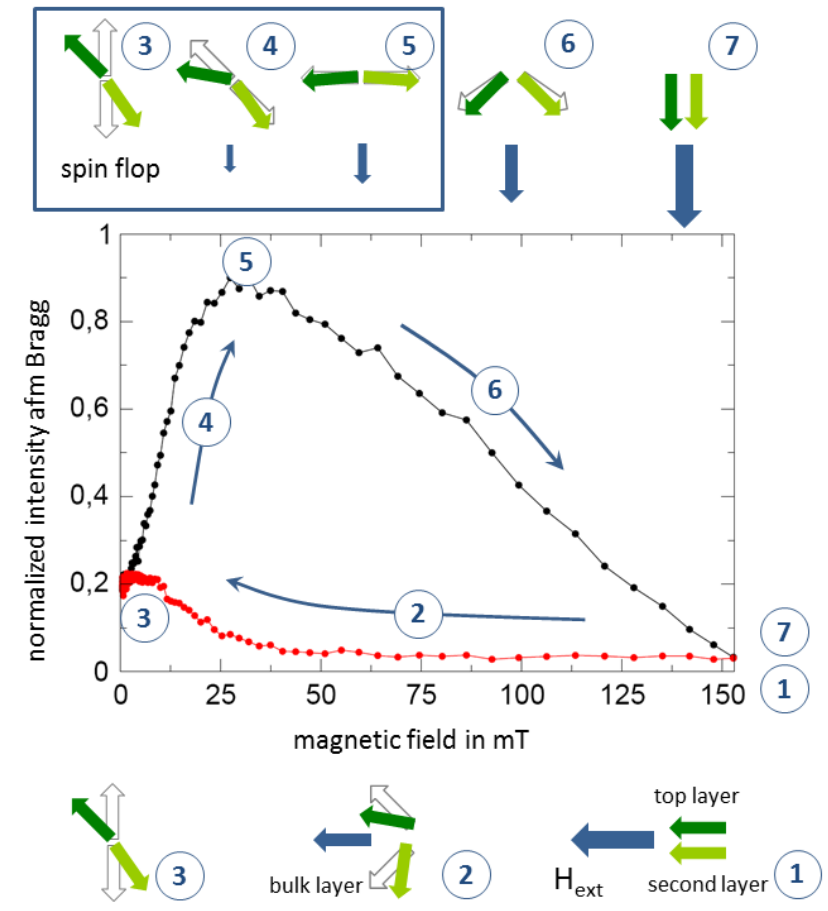

Fig. 7: Saturation behavior and spin flop transition in an antiferromagnetically coupled ${ }^{57} \mathrm{Fe} / \mathrm{Cr}$ multilayer monitored via the field dependent intensity of the first antiferromagnetic (afm) Bragg peak. The red curve shows the signal of the afm peak for a gradual decrease of the external magnetic field parallel to $k_{0}$. The black curve shows the signal for a subsequently applied increasing external magnetic field perpendicular to the beam which reveals a spin flop transition (rotation of the magnetic sublattices by $\pi / 2$ ) at low fields as well as the saturation behavior of the exchange coupled multilayer. A saturation field $B_{\text {sat }}$ of around $160 \mathrm{mT}$ can be identified. The green arrows indicate the magnetization direction of the topmost two ${ }^{57} \mathrm{Fe}$ layers, while the white arrows represent the magnetic moments of the layers deep inside the multilayer. In these sketches the photon wavevector $k_{0}$ is oriented horizontally.

easy axis of the spins structure in the antiferromagnetic ground state. The opposing Zeeman energy terms of the magnetic layers with even and odd numbers force the spin ensemble in the low field regime to perform a sudden magnetic reconfiguration by a rotation of $\pi / 2$ before the saturation regime starts [28]. This so called spin-flop phenomenon causes rather small changes in the magnetization signal of conventional experimental methods like MOKE and SQUID magnetometry [29] but results for the NFS technique in a drastic signal change of the nuclear superstructure reflection.

Fig. 7 shows the evolution of the first antiferromagnetic (half-order) Bragg peak of the ${ }^{57} \mathrm{Fe} / \mathrm{Cr}$ multilayer which was first magnetically saturated in a field parallel to the synchrotron beam. The stepwise decrease of the external field doesn't significantly increase the nuclear Bragg peak because the adjacent iron layers with an increasing canting angle exhibit no vectorial antiferromagnetic component parallel to the synchrotron beam. Close to remanence the spin configuration reaches the antiparallel ground state with a $180^{\circ}$ canting angle. The magnetization of the top iron layers exhibits a small deviation from that 
alignment due to the reduced symmetry at the surface. This leads to a small increase of the nuclear Bragg peak close to remanence (Fig. 7, red curve). Now we turn the direction of the external magnetic field by $90^{\circ}$ and slowly increase its magnitude while monitoring the intensity of the afm Bragg peak (black curve). The strong increase of the afm Bragg peak in the low field regime up to $25 \mathrm{mT}$ indicates the gradual evolution of the spin flop phase from the outer to the inner part of the multilayer (creation of a planar domain wall).
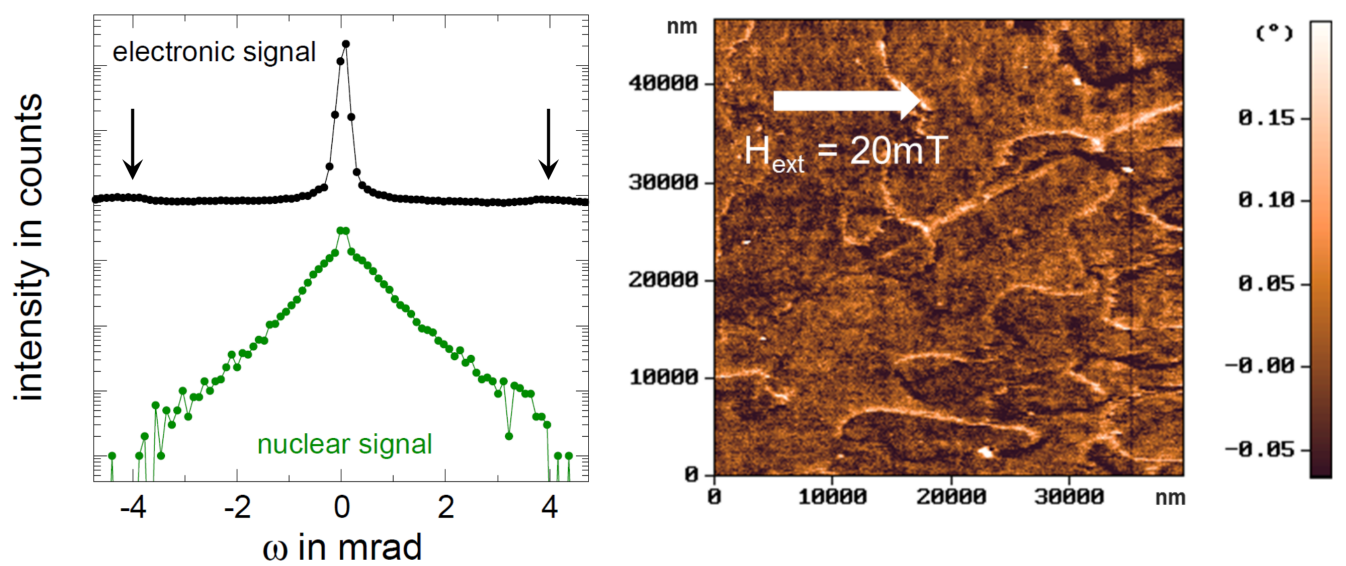

Fig. 8 Left: $\omega$-scan (rockingcurve) at the angular position of the first magnetic superstructure peak. The electronic signal is characterized by a strong specular reflection and a small intensity increase at the position of the Yoneda wings at \pm 4 mrad (arrows). The green curve shows the nuclear signal which can be used to investigate the lateral magnetic state of the iron layers. Here in the spin flop phase $\left(\mathrm{H}_{\mathrm{ext}}=20 \mathrm{mT}\right)$ an additional off-specular contribution can be detected which is due to lateral magnetic roughness or a domain structure of the system. An average magnetic correlation length (domain size) $\xi$ of around $6 \mu \mathrm{m}$ can be extracted from its width. Right: Surface sensitive magnetic force microscope image of the multilayer in the spin-flop phase. Pronounced domain walls that separate the antiparallel oriented domains are visible as bright horizontal bands. The dimension of the domains is consistent with the findings from the nuclear off-specular measurements.

\section{Domain formation in $\mathrm{Fe} / \mathrm{Cr}$ multilayers}

The antiferromagnetic interlayer coupling of the iron layers has a significant impact onto the lateral magnetic configuration. From surface sensitive measurements it is well known that ferromagnetically coupled magnetic multilayers exhibit a striped domain configuration while the antiferromagnetic coupling often bears a patch-like pattern [31]. Off-specular nuclear scans can now, very similar to those with polarized neutrons, be performed to study the domain configuration within the multilayer stack [32].

Fig. 8 shows the electronic and nuclear signal of a $\omega$-scan (rockingcurve) of the $\mathrm{Fe} / \mathrm{Cr}$ multilayer taken in the spin flop phase at an angular position of the first antiferromagnetic Bragg peak. While the signal in the electronic channel is characterized by a sharp specular reflection an additional very strong off-specular contribution can be seen in the nuclear signal. The angular width $\Delta \omega$ of this off-specular contribution is inversely proportional to the correlation length $\xi$ of the lateral magnetic roughness and identifies an average domain size of $6 \mu \mathrm{m}$. This value is very similar to that value detected with a surface sensitive magnetic force microscope on the multilayer in the identical magnetic configuration (Fig. 8b). 
These findings open the question of whether vertical magnetic stray fields of the magnetic domain walls induce a correlation of the lateral magnetic pattern through the multilayer stack. To investigate this we detect the degree of coherent interference of the diffuse nuclear signal via
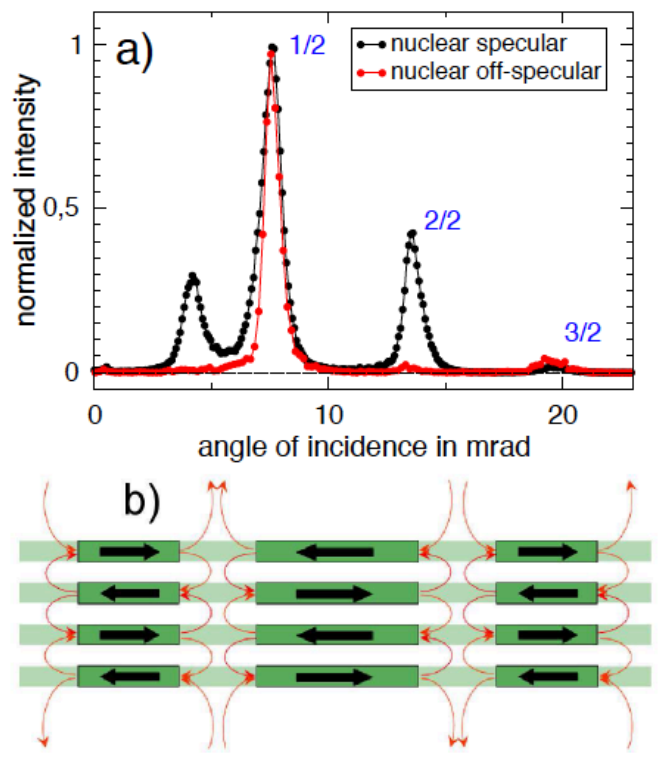

Figure 9: a) Nuclear specular and off-specular reflectivity of an antiferromagnetically coupled $57 \mathrm{Fe} / \mathrm{Cr}$ multilayer in the spin-flop phase $(\mathrm{Bext}=25 \mathrm{mT})$. A strong supression of the nuclear intensity is visible at the position of the structural Bragg peaks as well as at the critical angle. These findings indicate a very strong correlation of the lateral magnetic domain state (magnetic roughness) through the multilayer stack as pictured in b). This state is prefered due to minimization of the magnetic stray field energy (red lines).

an off-specular reflectivity (or $\theta-(2 \theta+\delta \theta)$ - scan). Fig. 9 shows the normalized specular and off-specular nuclear reflectivity of the multilayer in the spin flop phase $\left(\mathrm{H}_{\text {ext }}=20 \mathrm{mT}\right)$. A strong suppression of the off-specular signal at the position of the structural Bragg peak can be identified but antiferromagnetic off-specular Bragg peaks of unchanged width and relative strength can be seen. We can conclude that the diffuse nuclear signal interferes constructively at the angular position where every second magnetic layer is excited by an enhanced strength of the standing wave field but interferes destructively for a wave field that is exponentially damped within the multilayer stack. These findings assume a strong correlation of the lateral domain pattern through the multilayer stack (see Fig. 9b).

\section{Non-collinear coupling in Fe/Fe-Oxide multilayers}

As shown in the previous examples, nuclear resonant Bragg diffraction from single crystals and multilayers is very sensitive to magnetic superstructures that originate, e.g., from the moment canting between different magnetic sublattices. This has been applied recently to study the magnetic structure of Fe/Fe-oxide multilayers where a native Fe-oxide mediates a non-collinear coupling between the Fe layers [24,25].

Magnetic heterostructures that are formed of a magnetic material and its native oxide are relatively unexplored. Such layer systems were recently proposed as a new material with high magnetization and low electrical conductivity [26]. The magnetic coupling between two ferromagnetic layers separated by an ultrathin oxide layer exhibits remarkable properties, as revealed via in-situ nuclear resonant scattering during the growth of such heterostrucures. 
The beat pattern in Fig. 10a is characteristic for a parallel alignment of the Fe layer magnetization with $k_{0}$. This pattern does not change if the Fe layer is oxidized, as shown in Fig. 10b. Even though the oxide is separated by only $0.6 \mathrm{~nm}$ from the the probing ${ }^{57} \mathrm{Fe}$ layer, neither the magnitude of the hyperfine field nor its orientation are affected. This proves the very sharp boundary between the Fe metal and its native oxide. However, covering this bilayer with another iron layer that contains a ${ }^{57} \mathrm{Fe}$ probe layer leads to a significant change in the time spectrum as shown in Fig. 10c. The envelope of the beats is no longer exponential, but the intensity ratios of neighboring peaks exhibit an extra modulation. This can be attributed to a non-collinear alignment of the magnetic hyperfine fields in the two probe layers with the external magnetic field. From the data, one derives a coupling angle between the magnetic moments of these layers of $\theta_{c}=60^{\circ} \pm 5^{\circ}$, which is symmetric about the direction of the external field. In multilayers consisting of several Fe/Fe-oxide bilayers this type of coupling leads to the formation of two magnetic sublattices with a canting angle of $\theta_{\mathrm{c}}$, resulting in superstructure Bragg reflections for nuclear resonant scattering [24] and neutron scattering [27]. The origin for this coupling is the reduction of the multi-valent $\left(2^{+}, 3^{+}\right)$native Fe-oxide to single valent $\left(2^{+}\right) \mathrm{FeO}$ upon coverage with metallic Fe [33]. The ultrathin buried FeO layers exhibit an enhanced Neel temperature well above room temperature [34]. The exchange coupling between the Fe layers and the laterally inhomogeneous (rough) $\mathrm{FeO}$ layers leads to a spin frustration that results in canted moments rather than parallel or antiparallel moments between adjacent Fe layers [24].

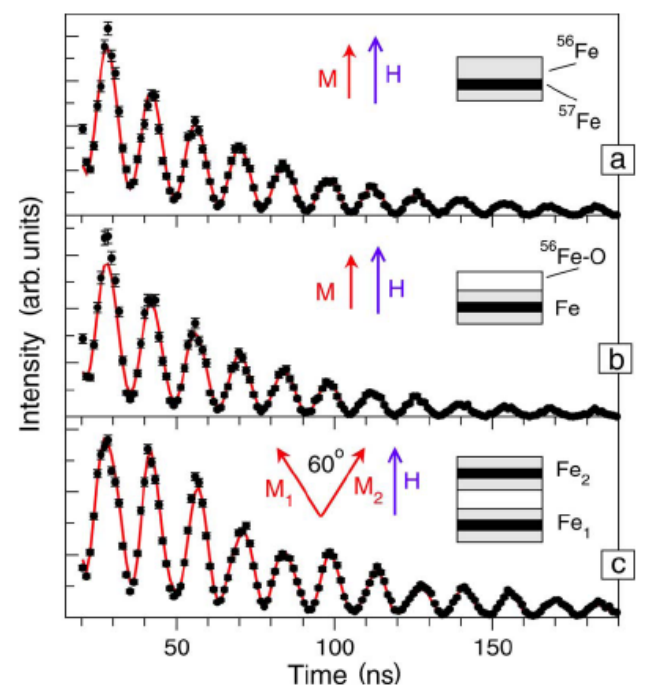

Fig. 10 Time spectra of NRS at three different stages of the growth of an $\mathrm{Fe} / \mathrm{Fe}$-oxide/ $\mathrm{Fe}$ trilayer. The solid lines are theoretical simulations from which the magnetization direction in the 57Fe probe layers relative to the external magnetic field of $H=70 \mathrm{mT}$ was deduced. The arrows show a top view of the relative orientation of the external field $\mathrm{H}$ and the sample magnetization $\mathrm{M}$. The wavevector $\mathrm{k}_{0}$ of the photons is parallel to $\mathrm{M}$.

\section{Conclusion and Outlook}

Nuclear resonant scattering of synchrotron radiation is a very useful technique to study the magnetic structure of low-dimensional systems. The isotopic specifity of the method facilitates the use of probe layers to selectively address specific sites in the sample, in particular interfaces between materials with different magnetic properties like ferromagnetic/antiferromagnetic, hard magnetic/soft magnetic, and magnetic/superconducting layers. Future applications will address time dependent and non-equilibrium phenomena as they occur, e.g., during phase transitions and magnetic switching processes. Such processes critically rely on the depth dependence of the magnetic 
structure as it changes as function of time. With micro- and nano-focused beams the spatial resolution can be significantly increased, e.g., by selecting individual magnetic layers in a cross-sectional transmission/reflection geometry. A very exciting perspective is the application of the method at future XFEL sources where it could help to understand the spatiotemporal evolution of ultrafast magnetic processes.

\section{Acknowledgements}

The experiments and studies reported here have been supported by the enthusiasm and endurance of many coworkers during all the years. It is a pleasure to acknowledge the contributions of Eberhard Burkel, Sebastien Couet, Thomas Diederich, Torsten Klein, Klaus Quast, Heiko Thomas and others to the various projects in thin-film magnetism. Particular thanks go to Rudolf Rüffer and his team at the beamlines ID18 and ID22N of the ESRF where all the nuclear resonant scattering experiments described here were performed.

\section{References}

[1] G. Schatz and A. Weidinger, Nuclear Condensed Matter Physics: Nuclear Methods and Applications, 2nd ed., Wiley, New York, 2006.

[2] R. Röhlsberger, Nuclear Condensed Matter Physics with Synchrotron Radiation: Basic Principles, Methodology and Applications, Springer Tracts in Modern Physics, Vol. 208, Springer, Berlin, 2005.

[3] S. L. Ruby, J. de Physique 35 (1974), C6-209 - C6-211.

[4] E. Gerdau, R. Rüffer, H. Winkler, W. Tolksdorf, C. P. Klages, and J. P. Hannon, Phys. Rev. Lett. 54 (1985), 835 - 838.

[5] J. B. Hastings, D. P. Siddons, U. van Bürck, R. Hollatz, and U. Bergmann, Phys. Rev. Lett. 66 (1991) $770-773$.

[6] E. Gerdau and H. de Waard, eds., Nuclear Resonant Scattering of Synchrotron Radiation, Part A, Hyperfine Interact. 123/124 (1999).

[7] J. P. Hannon and G. T. Trammell, Phys. Rev. 169 (1968) 315 - 329.

[8] J. P. Hannon and G. T. Trammell, Phys. Rev. 186 (1969) 306 - 325.

[9] R. Röhlsberger, Hyperfine Interact. 123/124 (1999) 301 - 325.

[10] D. Gibbs, D. R. Harshman, E. D. Isaacs, D. B. McWhan, D. Mills, and C. Vettier, Phys. Rev. Lett. 61 (1988), 1241-1244.

[11] J. P. Hannon, G. T. Trammell, M. Blume, and D. Gibbs, Phys. Rev. Lett. 61 (1988), 12451248.

[12] E. Gerdau and H. de Waard, eds., Nuclear Resonant Scattering of Synchrotron Radiation, Part B, Hyperfine Interact. 125 (2000).

[13] R. Röhlsberger, J. Bansmann, V. Senz, A. Bettac, K. L. Jonas, K. H. Meiwes- Broer, and O. Leupold, Phys. Rev. B 67 (2003) 245412. 
[14] C. L'abbé, J. Meersschaut, W. Sturhahn, J. S. Jiang, T. S. Toellner, E. E. Alp, and S. D. Bader, Phys. Rev. Lett. 93 (2004) 037201.

[15] K. Schlage, R. Röhlsberger, T. Klein, E. Burkel, C. Strohm, and R. Rüffer, New. J. Phys. 11 (2009) 013043.

[16] H. C. Wille, Yu. V. Shvyd'ko, E. E. Alp, H. D. Rüter, O. Leupold, I. Sergueev, R. Rüffer, A. Barla, and J. P. Sanchez, Europhys. Lett., 74 (2006) 170 - 176.

[17] H. C. Wille, R. P. Hermann, I. Sergueev, U. Pelzer, A. Möchel, T. Claudio, J. Persson, R. Rüffer, A. Said, and Yu. V. Shvyd'ko, Europhys. Lett. 91 (2010) 62001.

[18] R. Skomski and J. M. D. Coey, Phys. Rev. B 48 (1993) 15812 - 15816.

[19] E. F. Kneller and R. Hawig, IEEE Trans. Magn. 27 (1991) 3588 - 3600.

[20] E. E. Fullerton, J. S. Jiang, M. Grimsditch, C. H. Sowers, and S. D. Bader, Phys. Rev. B 58 (1998) 12193 - 12200.

[21] J. S. Jiang, H. G. Kaper, and G. K. Leaf, Discrete Contin. Dynam. Syst. Ser. B 1 (2001) 219232.

[22] R. Röhlsberger, H. Thomas, K. Schlage, E. Burkel, O. Leupold, and R. Rüffer, Phys. Rev. Lett. 89 (2002) 237201.

[23] T. S. Toellner, W. Sturhahn, R. Röhlsberger, E. E. Alp, C. H. Sowers, and E. E. Fullerton Phys. Rev. Lett. 74 (1995) 3475 - 3478.

[24] Th. Diederich, S. Couet, and R. Röhlsberger, Phys. Rev. B 76 (2007) 054401.

[25] S. Couet, Th. Diederich, S. Stankov, K. Schlage, T. Slezak, R. Rüffer, J. Korecki, and R. Röhlsberger, Appl. Phys. Lett. 94 (2009) 162501.

[26] G. S. D. Beach, F. T. Parker, David J. Smith, P. A. Crozier, and A. E. Berkowitz, Phys. Rev. Lett. 91 (2003) 267201.

[27] S. Couet, K. Schlage, Th. Diederich, R. Rüffer, K. Theis-Bröhl, B. P. Toperverg, K. Zhernenkov, H. Zabel, and R. Röhlsberger, New J. Phys. 11 (2009) 013038.

[28] U. K. Rößler and A. N. Bogdanov, Phys. Stat. Sol. (c) 1 (2004) 3297.

[29] S. G. Velthuis, J. S. Jiang, S. D. Bader, and G. P. Felcher, Phy. Rev. Lett. 89 (2002) 127203.

[30] V. Lauter-Pasyuk, H. J. Lauter, B. P. Toperveg, L. Romashev, and V. Ustinov, Phys. Rev. Lett. 89 (2002) 167203.

[31] M. Rührig, Phys. Stat. Sol. (a) 125 (1991) 635. 
[32] D. L. Nagy, L. Bottyan, B. Croonenborghs, L. Deak, B. Degroote, J. Dekoster, H. J. Lauter, V. Lauter-Pasyuk, O. Leupold, M. Major, J. Meersschaut, O. Nokinov, A. Petrenko, R. Rüffer, H. Spiering, and E. Szilagyi, Phys. Rev. Lett. 88 (2002) 151803.

[33] S. Couet, K. Schlage, K. Saksl and R. Röhlsberger, Phys. Rev. Lett. 101 (2008) 056101.

[34] S. Couet, K. Schlage, R. Rüffer, S. Stankov, Th. Diederich, B. Laenens, and R. Röhlsberger, Phys. Rev. Lett. 103 (2009) 097201.

[35] P. Grünberg, R. Schreiber, Y. Pang, M.B. Brodsky and H. Sowers, Phys. Rev. Lett. 57, 2442-2445 (1986)

[36] P. Bruno and C. Chappert, Phys. Rev. Lett. 67, 1602-1605 (1991) 